\title{
Cosmoharmonic principles of environmental safety
}

\author{
Mariana Basilaia $^{1,}$, Irina Bogdanova $^{1}$, and Olga Dymnikova $^{1}$ \\ ${ }^{1}$ Don State Technical University, Gagarin sq., 1, Rostov-on-Don, 344000, Russia
}

\begin{abstract}
In the course of globalization the humanity tends to critically regard the traditional ideals of the civilization evolution. It is in the arduous quest for rational routes of advancement, novel values capable of untying antagonisms and protecting the civilization from the global disaster. Any philosophical system is a resolution of the time's antagonisms. Philosophical comprehension of the environmental antagonism and way of ascending into the noosphere is attainable through a concept of cosmoharmony resting upon dialectical uniformity of fundamental and philosophical knowledge giving rise to a new world outlook.
\end{abstract}

\section{Introduction}

The informational era with its technologies, global interrelationships and their consequences has transformed this world beyond recognition. The humanity has not yet had much time enough to adapt itself for booming information technologies which have modified people's conventional concepts of themselves, of their interrelation among themselves and with their environment, of the development of society and culture. Nowadays nearly all domains of human life are found in the grip of the global computer network Internet, its new principles organizing our existence: network, virtual, interactive. Consequently, there have arisen such new phenomena as the digital economy, distant learning, electronic management and control, etc. conducive to formation of virtual communities, embracing a vast number of people of different continents devoted to a certain idea.

New informational openness and technological revolution have stimulated the intensified synthesis of national economies into a united worldwide system which gave rise to communicational convergence of industrial nations, and cleared the way for an avalanche of goods and capital to qualitatively change the nature of business.

It is essentially important that, with all this going on, the modern society's object of production has been changed and still changing. Whereas through the entire history of mankind, the principal basis for existence was natural resources, then a new phenomenon is showing up now. Since up-to-date information technologies have stimulated new, more lucrative tenors in business to arise, the problem of nature transformation has been amplified with a factor of changes in human consciousness both individual and collective.

\footnotetext{
*Corresponding author: mariana3061@mail.ru
} 
Both of these factors, as everybody knows, are of the first priority in the processes of catastrophic exacerbation of global ecological crisis.

Hence, the globalization under current objective conditions determined by market rather than governmental forces contains a dangerous potential of the ecological catastrophe expansion. In this connection, the already acute problem of environmental safety is becoming the dominating world problem [1].

\section{Materials and Methods}

In the planet's ecological processes the mechanical, chemical and biological natural phenomena almost completely add up to a ceaseless conversion of energy. Any complex system, be it artificial or natural, is strictly subjected to the essential laws of nature having a universal significance. The biosphere, or global ecosystem, defying these laws or being exposed to such conditions in which the laws of nature would be violated, is doomed to destruction.

It is natural that the idea of physics' new era was starting to take a shape with emergence of the energy conservation law. It is this law, as we understand, which is supposed to bring us, in the long run, to a wide disclosure, comprehension and extension of cosmoharmony and attainment of environmental equilibrium. That is the reason why we should refresh some basic concepts of thermodynamics to sustain a logical connection with the environmental safety.

Energy in ecological systems (as any energy at large) is defined as an ability to execute the work, in other words, the ecosystem's energy is perceived as a sum of abilities of this system to actions designating its vital activity. The first law of thermodynamics, or the energy conservation law, maintains that energy is capable of converting from one form to another, but it remains to be of an invariable value, i.e. it neither disappears nor is reborn anew. The second law of thermodynamics, or the law of entropy, maintains that processes connected with energy conversion can occur spontaneously provided only that the energy is converted from its concentrated form into a dispersed one (becomes degraded); or in other words: since some portion of energy is always dissipated in the form of inapplicable thermal energy, the efficiency of spontaneous conversion of kinetic energy (e.g., sunlight) into potential one (e.g., energy of protoplasm chemical compounds) is always below 100 percent.

\section{Results}

While studying complex processes related to energy dissipation, that is, to universal energy tending towards ubiquitous equilibrium, denoted as energy dissipation, Clausius coins the term entropy by which he understands the value of already converted energy, i.e. energy exposed to such conditions that it cannot be inversely converted any longer (e.g., heat diffused in outer space). Hence we can better understand basic concepts of Clausius: 1) energy of the universe is constant; 2) entropy of the universe tends to reach maximum [2]. Thus the sum of energies of the entire Universe is invariable, but it is not the case for various parts of the Universe, ecosystems among them. At the same time, though according to the first law of thermodynamics, energy is neither created, nor annihilated, none the less, all the variety of life manifestations features continuous transformations of energy. Life of living organisms is sustained not by energy per se, but by its transformations (a process).

Now we turn to the subject of interest to us, to absolutely real events but quite contrary to optimistic aspirations for the trend of ecological stability.

Ecological systems, their interrelations globally ensuring conditions for life on our planet are closely connected with those types of convertible energy dissipated on the Earth. 
Here are the basic ones: energy of the Earth's rotation round the Sun and its own axis; the Earth's internal heat manifested in volcano eruptions, earthquakes (forces of sudden, perturbation, destructive effect); air motion (wind); chemical agent and compounds; power of water streams and water movements; energy events linked to anthroposphere/technosphere activity.

Presently an ever growing role in the balance of biosphere's energy budget becomes to be played by an energy component introduced with activity of human civilization. Naturally this information-energy constituent of the biosphere's vital activity being relatively new for the planet has never been taken into account of the Earth's stable evolution and its vital energy balance. However real processes of "the technical progress" of the civilization have actually never been practically associated on a system level with global environmental events for the purpose of sustaining stability of the planet's life-supporting processes. Day by day consumer wants of the so-called "civilized" nations are plainly becoming runaway to gain super-profits.

Temptation of consumerism carries away today's civilization (at least a huge part of it) into infinite abyss of illusive fleshly "delights" bearing a stamp of earthliness and unsociability, condemning to destruction everything which is a backbone of human life and entire biosphere: nature, harmony of the Universe's earthly structures. And everything inducing the destruction of the civilization's foundations is naturally reflected in our consciousness to retain all realities and mould our consciousness as being apt to a fleshlyconsuming, fatal, obstinate illness [3].

It is significant that the term "krisis" as translated from the Greek means a resolution, verdict, resolute outcome. Thus the notion of ecological crisis quite clearly and adequately develops the implication of the verdict and resolute outcome of covetous, irregular, infiniteprofit-minded destruction of nature. And this contradicts the true values of society's material and spiritual life and adherence to its moral tenets.

When among global disasters reported in the mankind's chronicles, we happen to find data on collapse of the continent of Atlantis, on abrupt large-scale devastations followed by tragic aftermaths (floods, earthquakes, eruptions, etc.), we tend to unwittingly contemplate on the fact that such kind of phenomena have already begun manifesting themselves with unprecedented frequency since the second half of the $20^{\text {th }}$ century in exceptionally intensive manner. As this takes place, experience leads us to conclude that tragic consequences for the society add up not only to casualties and property damages, but they can occur in the form of severe psychic trauma (for both: individuals and public consciousness involved in global disasters). It leads to dramatic changes in consciousness followed by deformation of characters, principles, views and foundations designating their lifestyle. It stands to reason that all this was reflected in views of thinkers pertaining to different epochs.

\section{Discussion}

In completive decades of the last $20^{\text {th }}$ century power engineering as a key factor of any development was paid much attention in scientific publications both in this country and abroad, specifically on fuel and energy crisis related to firewood (Eckolm, 1975); "Revision of nuclear power: Chernobyl aftermaths" (Flavin, 1987). However no adequate resonance within the public was observed. Instead we had a sort of flings at extremes: the impendent wood crisis in developing countries and constant ups and downs in rude oil prices in the world market; in mid 70s the world was swept over with technocratic euphoria fueled by expectations that APP would resolve all the power problems.

So what we observe now is plunder of natural life-securing energy and simultaneous amassing of negative destructive energy, which uncontrolled effect can hardly be foreseen. By the plunder of ecological energy we understand people's actions inducing dissipation 
(degradation) of global ecosystems resulting in a diminishing budget of the biosphere's convertible energy. The notion of natural power plundering is related to the mankind's activity, for apart from such activities a continuous outage of energy into ambient space occurs, i.e. natural straggling. Plunder of natural energy implies intensification of this outage through people's specific activity resulted in environmental misbalance with its ensuing negative consequences including those in respect to human society.

Among enterprises which are severely hazardous to environmental safety are, e.g., all major power corporations and other ventures directly linked to nuclear, petroleum, natural gas, coal and other industries generating super-contaminants (i.e. emissions incapable of being purified by own nature's strength), radiation pollution of regions, destruction of the planet's flora and fauna. Data on the use of petroleum products on a global scale confirms the consumer attitude of nature, leading to degradation and disruption of the natural balance (Table 1).

Table 1- World oil consumption by major regions and countries (million tons per year)

\begin{tabular}{|l|l|l|l|l|l|l|}
\hline & $\mathbf{2 0 1 3}$ & $\mathbf{2 0 1 4}$ & $\mathbf{2 0 1 5}$ & $\mathbf{2 0 1 6}$ & $\mathbf{2 0 1 7}$ & $\mathbf{2 0 1 8}$ \\
\hline World consumption & $\mathbf{4 5 4 2}$ & $\mathbf{4 5 8 8}$ & $\mathbf{4 6 3 7}$ & $\mathbf{4 6 8 9}$ & $\mathbf{4 7 4 5}$ & $\mathbf{4 8 0 5}$ \\
& & & & & & \\
\hline USA & 959,9 & 964,6 & 981,0 & 985,7 & 991,8 & 997,9 \\
\hline Canada & 121,1 & 119,4 & 118,7 & 118,7 & 119,0 & 119,5 \\
\hline Europe & 678,0 & 669,7 & 664,2 & 659,0 & 655,7 & 654,4 \\
\hline Japan & 225,7 & 215,5 & 208,7 & 205,8 & 203,3 & 201,2 \\
\hline China & 512,9 & 531,3 & 547,6 & 564,7 & 582,2 & 599,7 \\
\hline Asian countries (other) & 570,1 & 581,0 & 591,6 & 607,6 & 622,8 & 637,1 \\
\hline Countries of the former USSR & 239,3 & 242,8 & 233,7 & 229,5 & 227,2 & 228,3 \\
\hline Russia & 133,2 & 137,7 & 130,5 & 132,5 & 137,0 & 138,0 \\
\hline
\end{tabular}

The environmental safety is endangered with wars with all the subsequences catastrophic for the planet's ecology but again overland, in water and in the atmosphere. All these phenomena are just a mere robbery of natural/environmental energy available at the disposal of the life sphere - biosphere. No doubt that an opportunity of energy distribution more reasonable for nature and advantageous for man to a great extent is in human hands.

V.I. Vernadsky was one of the first ones who predicted that the mankind was becoming a powerful geological force, and that we were going through a new geological evolutional transformation of the biosphere and entering the noosphere. For now the findings of scientific analysis attest that the ecological crisis of environment caused by human activity poses a threat not only for wellbeing, but for the world community's survival as such.

And despite a number of initiatives by the International Geosphere/Biosphere Program (IGBP) and other international programs, environmental instability and processes of plundering the eco/energy resource keep on building up. In this connection the World Watch Institute (WWI) has evolved a concept of transition to the path of stable ecoeconomical development, which burden was the so-called ecological deficit. When impact on natural ecosystems exceeds their productive capabilities, ecological deficit arises. As a matter of fact, it is a satisfaction of today's needs at the expense of future resources, i.e. exploitation of the future which is unacceptable neither from moral considerations, nor 
from material ones. The WWI pointed to the similarity of causes generating ecological and economic deficits. But it should not be overlooked that whereas economic deficits make themselves felt instantaneously, ecological deficits have delayed-action repercussions, and more often they do when ecology is found in a state of crisis, when renewable natural resources become nonrenewable.

Having found itself in a state of ecological deficit, the society, shaken by catastrophes, sets about searching for a universal solution of the way-out-of-crisis problem. Today it is obvious that programs and good intentions expounded in eco-saving documents and literary sources have so low efficiency that the level of ecological stability, the planet's safety is permanently lowering itself. Ecological misbalance issues the formidable challenge for the mankind: to ensure environmental safety and the community's survival.

The mankind is faced with the "to be or not to be" dilemma exacting a prompt solution. However the progress of social environment stimulated mainly by the activity which primal vector are consumer's ambitions and imaginary comfort of material origin, has led us up a blind alley of ecological misbalance. Such situation poses several pressing questions for the mankind: is there any factor capable of balancing the society's prevailing material and economic ambitions? What is the implication of the activity which vector of a new direction will create a resultant force of evolution leading not to a deadlock of ecological destruction, but towards stable noospherical prospects? In what way should a philosopher expose himself to fusion of our time's specific antagonisms in their basics and how is this issue to be developed in one's scientific work?

Quest for the universal outlet from the negative process of development is an objective in common for the entire mankind without any borders and social divisions.

Such universal process could be improvement of the civilization's activity and lifestyle based on principles of actual harmonious unity with nature, with entire ambient planetary and cosmic medium, the process of Cosmoharmony.

This is a universal holistic process keeping extremes in unity and self-organizing everything on the basis of numerical relations in the Universe and in microscopic and macroscopic spheres. The process of cosmoharmony is conditioned by energy constancy in homogeneous space and infinite kinematical change in time. The outlook for the future is that we set for ourselves a task to not only conceptually describe these phenomena in philosophical terms, but to endeavor to bring the findings of multiyear investigations closer to real tasks of the cosmoharmony ideas' evolution from the position of fundamental approaches and casting their exploratory models [4]. On the other hand, present-day investigations in the field of psychology bring us on the verge of getting insight into depths of the crisis psychological foundation - the people's ecological awareness targeted at the decisive human factor of environmental safety [5].

Logos of cosmoharmony is raised before us by time itself, by the Universe itself. It is essential to have a cosmo-harmonic interaction of all-to-all within the Chaos - Order system: Man and Nature, living and inanimate matter, substance and spirit, science and religion, etc.

Cosmoharmony, in our view, is not about the world's static beauty, even more so it is not a structure. This is a process of interaction of all living entities in a harmonious unity. It is an inception of a new knowledge, a new science of the planet's environmental safety. Harmony will save this world. It is harmony that will spring the beauty which apparently Dostoevsky had in mind saying about the role of beauty in salvation of this world. Cosmoharmony is the fundamental basis of the Universe functioning principles evolving from a singularity to infinity and backwards on the principle of pendulum swinging within the Chaos - Order medium from minimum entropy to maximum one and vice versa. This notion contains a key to the control of development processes geared to "the mechanism of celestial infinity". Let us try to answer the question raised by ourselves above: where is the 
second balancing vector to generate the resultant of salvation to help out of the catastrophic deadlock?

In order to withhold the biosphere degradation and guard the mankind against the tragic ecological crisis, apart from novel techniques, a new highly cultured ecology-sensitive consciousness is deadly needed; let us call it a cosmo-harmonious consciousness. However, apparently the ecological knowledge and even high culture might still be not enough. The mankind would require realization of a supreme reality and attaining such a world outlook that could blend with the Universe's harmonious (though suffused with dramatic rhythms) fundamental substance comprising a diversity of phenomena, many of which are still beyond our grasp. At the same time, it is amply evident that the world thinkers throughout millennia aspired to grasp the fact that fluctuation and instability are inherent to evolution of everything in this world: both man, and nature.

F. Capra's intrinsic merit is his efforts directed at exploration of scientific strata in ancient conceives' works. It was pretty hard for a westerner to comprehend and share the idea (particularly in pre-Hegel period of philosophical thought development) of intrinsic unity of all opposites. However, the oriental philosophy, its very foundation is underlain with the conception of unity and interrelation of opposites. In the East they considered all changes in nature (including those in human physiology) as a dynamic interaction of the two opposites: Yin and Yang. Orientalists apply the philosophy of such orientation for comparison, maintaining that Chinese philosophers "have developed an approach, deeply scientific in its essence, and it was only their strong distrust towards the analytical method that prevented them from evolving truly scientific theories", and still their reflective observations of nature coupled with profound intuition brought oriental wise men "to amazing discoveries which validity is verified with contemporary scientific theories"[6]. The aforementioned work's author, F. Capra draws an interesting parallel between the world outlooks of two thinkers of the $6^{\text {th }}$ century B.C. Concurrently with Lao-tzu's creative work, who found followers in China and advocated concepts of perpetual changes ("Everything flows") and their cyclic nature, in Greece Heraclites of Efes was describing the world structure with "ever-burning flame, now kindled, now quenched". Ideas personified in Heraclites' characters, and the Chinese philosopher's conceptions of cyclic alternation of Yin and Yang feature a substantial affinity of these thinkers' world outlooks.

Hence thinkers of different philosophical schools being absolutely independent arrived at the conclusion that changes were a dynamic interchange of opposites, and all the opposites were polar, and therefore unified. As regards man as such, for him in the dynamic interchange of two opposites in the first instance concepts of good and evil correlated as Yin and Yang come out [6].

As for the complex system of Man - Nature - Cosmos or Man - Biosphere - Cosmos, the issue of universal solution of the task of bringing the civilization out of ecological crisis might have real optimistic prospects. Reliability of such a system and hence its environmental safety will be certainly higher if we succeed in harmonious synthesizing a material and spiritual constituents of this world.

Interconnection of the two fundamental spheres within a single system, their harmonious integrity and complementary functional non-detachability might become a cosmo-harmonic guarantor of the global environmental safety.

However, optimism of such a solution of the problem of environmental safety is associated with an extreme complexity. The pressing ambience for the nature through continuous anthropogenic and technically-induced stresses must be replaced with the absolutely new ambience: that one of coordination, harmonious interrelation of man and nature, ambience of irreconcilable resistance to plundering and destruction of the planet's information/energy resources. It is exceedingly important that man's new world, his outlook and lifestyle were endowed with tolerance, that is, a moral quality featuring attitude 
to an individual as to an equally worthy person (irrespective of national, racial, cultural, religious and other distinctions).

Any philosophical system embodies resolution of its time's antagonisms. Cosmoharmony is targeted at resolution of contradictions between Nature and Man; it is an Earthly or Biospherical component of the universe-wide cosmoharmony. But so far in present-day situation, consumer's fervors and the mankind's forcible activities might lead to unpredictable scenarios of evolution, and are fraught with a global catastrophe. That is the reason why an important step to take should be a practical constituent of the global tasks solution.

The basic antagonism of our time should be resolved in the thick of the world community's life in a practical sphere, and this practice should provide access to global values.

Humanity's life, the community's life can resolve the basic antagonism of our time only through elevation of people's spirituality, their spiritual culture up to the level of profound realization of essential and firm orientation at the creative lifestyle with respect to the habitat.

Just a mere anticipation of the new world, we are longing for, nor matter in what way we are going to designate it: a noosphere or postindustrial renaissance, will be futile without a painstaking, goal-seeking work. A mere recognition of various methods of consideration (as was the case after the international congress in Rio de Janeiro, 1992) can hardly provide a hands-on solution of the global task. Broadmindedness turned out to be a poor substitute for essential creative work and practical workmanship in global terms. Recognition of acceptability of alternative grounds, techniques and forecasts in respect of the new worlds' creation is still not enough to contrive any scientific theory or philosophical system.

Presently, the time's ecological antagonism is relatively recognized but absolutely not coped with. It is expressly evidenced by the fact that regional profoundly mercenary contradictions hold sway over humanity, increasingly aggravating the ecological deficit. In this situation, in the process of hard working directed at creation of foundations for the new world, the noospherical world dreamt of by V.I.Vernadsky, the world of ecological renaissance of the $21^{\text {st }}$ century, one can hardly overestimate the global role of philosophy.

Ecological stability, safety of evolution enabling humanity to survive in the context of the Universe's formidable challenges is our destiny. It is what in no way we can evade. Whether we are upset as we come upon some knowledge while still coming across mysteries, or to the contrary we enjoy this paradoxical situation, depends on the observer's personality. And every observer is supposed to have some grounds, i.e. a judgment or conception to be a source of some reality. This idea was also rendered in a highly methodical and world-outlook-wise work by Heidegger: "Wherever you shoot a look, laments Heidegger, discussion about The Principle of Reason (Der Satz vom Grund) from the first steps sinks into darkness" [7].

Ostensibly, that is the way this world is structured: clear things need obscure ones, and light needs shade, otherwise what would we need to clarify? Goethe in his time cited quite an accurate phrase belonged to Johann Georg Hamann: "Clarity is a proper partition of light and shade". However, if we turn to human cosmoharmony, being a separate new notion, then from this perspective we can see that light dissipates dark, and under the same cosmic law but already not in the physical, but rather in the spiritual world: love dispels evil. Our knowledge and cultivation of loving strength towards visual environment enable us to surmount all destructive consequences of aggressive hatred.

Let us discern this partition of light and shade in the modern biosphere's ecological processes. The ecological sustainability and ensuing environmental safety of our civilization, these two capacious notions under the conditions of booming techno-sphere are 
placed in the forefront of actual problems of humanity's survival. The biosphere and techno-sphere incorporated by man thereto are ever-advancing global systems. Products of the techno-sphere and its interaction with the biosphere provide humanity's life with auspicious conditions. It is the interaction of these two global systems where relation of light and shades is found. Each of these complex systems is noted for its own growth specificity, complexity and diversity of its constituents. The complication is that while making the global unity complete, they are deeply confronted. At present time humanity's destiny is correlated to and extremely dependent on ecological processes taking place within these systems. In this connection the most cardinal problem of modern humanity is brought to the forefront: ensuring such a nature and dynamic state of the community's technologies development and complex ecosystems that would foster sustainable and safe vital activity for humanity and all living beings on the Earth .

While entering the cosmoharmonic sphere of thinking, we begin feeling a necessity of expanding the action field for the knowledge available. Regarding the complex nonlinear Man - Biosphere - Cosmos system, we deem it expedient to try to combine philosophical reflections with fundamental knowledge of the world ecosystems and human factor within their medium.

Everything linked to cosmoharmony is directly related to global elements and ecosystems securing the Earth's biosphere vital activity. And analysis and even a brief rating of the world ecosystems' significance in evolution of our planet, being a complex multifaceted issue, require a fundamental scientific basis.

Principles of fundamental substantiations in the philosophy of global environmental safety are thoroughly examined in exploring some new theoretical trends [4]. The basic motives of these principles are as follows.

The language of abstractions has never been alien to philosophy, but resorting to, let us say, mathematics was rear. But now a question arises: aren't common and productive dialogs between them (even though quite inconsistent) the catalysts of great progress propelling us towards the truth? [8].

Let us recall Kant's merit rating of mathematical thinking, which "trains the mind to detect the nature's order and correctness in the sublime and little, as well as amazing unification of all its driving forces and thus gives the mind cause and stimulus for application going beyond any experience, and, besides, it gives philosophy, dealing with these issues, an excellent material upholding its investigations to the extent allowed by their character through appropriate contemplations" [9].

Interdependence of philosophy and abstract sciences, mathematics in the first place, is one of the scientific foundations of the theory related to curbing the ecological crisis. Each and every of these abstract sciences is endowed with its own irreplaceable complementary forces, knowledge and culture of perceiving the world. Herewith there exemplified an importance of such a big philosophical step as introduction of the principle of complementarity by Niels Bohr.

Manifestation of the complementarity principle in a dialectical unity of fundamental and philosophical knowledge presenting a dual integrity of global cosmoharmony opens up a new page in the creative search for a numerical degree of harmonious combination of contradictory manifestations of the material and spiritual worlds. It is this type of harmony originated from the laws of Universe and its constants is capable of generating natural conditions under which the unity and fight of opposites might take place in the framework of a productive process, rather than a destructive one [10].

\section{Conclusions}


Research which should provide not only explanations, but change the problem situation, must be initiated with a clear and substantiated specification of initial conditions. The initial conditions of the techno-sphere's developing processes are subordinate to differential laws and linked to a notion of objective which stands out as a transition from a forecast to a plan, from statement to realization of a previously arisen image or model. The initial conditions of global catastrophe are connected with inception of nonlinear processes giving rise to dangerous factors (e.g. a major disaster at APP or any ecological catastrophe on a continental scale) that under certain conditions result in a devastating catastrophe. The initial conditions integrate factors of the biosphere and man, concrete human activity, and taken decisions into a single system. At present a human factor holds the lead and determines the event's outcome where the problem relates to the techno-sphere and anthropogenic causes. In this context the Inception harbors a certain causal task. What brought a man who has taken this or that wrong decision to a fatal blunder? What are the reasons that impelled the operator to act inaccurately, erroneously, although there are precise instructions, and the operator is duly trained in terms of professional skills? A human factor (lately quite frequently referred to while investigating the causes of catastrophes) appears to be an exceedingly complex category, and this issue spurs up to more specific research.

To attain a higher stage of fundamentally new informational perfection ensuring an interacting harmony between man and nature, to attain the environmental safety offering the challenge of the mankind's further advancement, we need new knowledge embracing the new world perception based on a systemic approach and achievements of the globalization's spiritual values that guide us towards a new cosmoharmonic world outlook.

\section{References}

1. A. Chumakov, A. Ioseliani, Philosophical problems of globalization, 172 (2015)

2. E. Teterina, S. Piskorskaya, Actual problems of aviation and asronautics, 2 (7), 271$272(2011)$

3. M. Basilaia, The need to reduce environmental hazard as an imperative of the global world order (philosophical analysis), 338 (2010)

4. A. Astvatsaturov, European Applied Sciences, 7, 63-67 (2013)

5. M. Basilaia, Vestnik of DSTU, 5 (48), 792-797 (2010)

6. I. Abylgaziev, I. Ilyin, The Century of Globalization, 2 (4), 40-60 (2009)

7. A. Astvatsaturov, M. Basilaia, T. Tyurina, Perm University Herald. Series "Philosophy. Psychology. Sociology», 3 (23), 57-64, (2015)

8. A. Astvatsaturov, B. Meskhi, Science almanac of Black Sea region countries, 27, 15-18 (2015)

9. T. Leshkevich, State and Municipal Management. Scholar Notes, 3, 106-113, (2013)

10. A. Astvatsaturov, Oxford Philosophical Society, 33, 4-8 (2011) 